\section{Can ultrasound of the major salivary glands assess histopathological changes induced by treatment with rituximab in primary Sjögren's syndrome?}

With great interest we have read the recent publication by Fisher et $a l^{1}{ }^{1}$ entitled 'Effect of rituximab on a salivary gland ultrasound score in primary Sjögren's syndrome: results of the TRACTISS randomised double-blind multicenter substudy' in which the authors demonstrated a significant improvement in total ultrasound score (TUS) after rituximab treatment compared with placebo at weeks 16 and 48 in 52 patients with primary Sjögren's syndrome (pSS).

We and others have shown that treatment with rituximab, a chimeric anti-CD20 monoclonal antibody, affects the histopathology of the salivary glands and results in a decrease in area of lymphocytic infiltrate in the labial and parotid glands. ${ }^{2-5}$ This reduction in infiltrated area, that is, up to $50 \%$, is mainly caused by $\mathrm{B}$ cell depletion. ${ }^{2}$ Rituximab treatment also causes a significant loss of germinal centres. ${ }^{2}$ Interestingly, rituximab treatment also leads to a significant restoration of the ductal epithelium glandular tissue itself. This is illustrated by a significant decrease in number and severity of lymphoepithelial lesions (LELs) in parotid gland tissue 12 weeks after rituximab treatment. ${ }^{26}$ The normalisation of the epithelium appears to be a direct consequence of depletion of intraepithelial B cells. ${ }^{7}$

Significant improvement of the parotid gland ultrasound score of patients with pSS has been observed before in another randomised controlled trial (RCT) with rituximab, the Tolerance and Efficacy of Rituximab in Primary Sjogren's Syndrome (TEARS) study. ${ }^{8}$ This is an RCT with rituximab versus placebo, performed by a French group. Together with the improvement of the histopathology of the gland, it might not be a surprise that Fisher et al ${ }^{1}$ also observed a significant improvement in TUS. This newly developed scoring system is composed of five domains: echogenicity, consistency, definition, glands involved and hypoechoic foci size. In the study by Fisher et $a l^{1}$, the difference in TUS between rituximab and placebo-treated patients was only attributed to the 'definition' domain. This domain addresses the visibility of the salivary gland posterior border. Other studies have shown that this domain is hard to assess, ${ }^{19}$ which is also supported by the accompanying low interobserver reliability. ${ }^{10} 11$ None of the other components showed a significant improvement at weeks 16 and 48 after rituximab treatment in patients with pSS. ${ }^{1}$ In a previous study, we have shown that the presence of hypoechogenic areas is the most important component in the evaluation of pSS. ${ }^{12}$ Hypoechogenic areas contribute to several domains scored when using the ultrasound scoring system applied by Fisher et al, ${ }^{1}$ which include echogenicity, consistency and hypoechoic foci size. Although no significant improvement was found in these ultrasonographic components, there seems to be a tendency that the consistency domain does somewhat improve after rituximab treatment. ${ }^{1}$ On the basis of the observed histopathological changes seen in parotid glands after rituximab treatment, however, it might have been expected that more domains of the TUS would have been improved.

The question becomes now why Fisher et al, ${ }^{1}$ in our opinion, observe only minimal changes in ultrasound score after rituximab treatment. First, as long as we do not exactly know which histopathological parameters in salivary glands are being measured by ultrasound, it is hard to understand the effects of treatment on the ultrasound scores. While in the TEARS study, a good association between focus score in the labial gland biopsy and their ultrasound score was found, our group found only fair to moderate associations between the Hocevar ultrasound score ${ }^{13}$ and focus score in parotid and labial salivary gland biopsies, respectively. ${ }^{14} 15$ The focus score is not appropriate to measure the local effect of rituximab on the periductal lymphocytic infiltration. ${ }^{2}$ Moreover, a focus score does not reflect the size of the infiltrate and the inflamed area can be considerably reduced as a consequence of treatment, while the number of foci does not or only slightly decrease. Indeed, after rituximab treatment, the focus score was not altered, although there was a reduction in the size of the periductal infiltrate. ${ }^{2}$ Taking this limitation of the focus score into account, other measurements, such as the relative area of infiltrating CD $45+$ cells, the number of B cells $/ \mathrm{mm}^{2}$ of glandular parenchyma, the presence and stage of LELs as well as the presence of germinal centres, more accurately define the magnitude of the inflammatory status of the gland. ${ }^{2}$ Since these histopathological features are too small to be represented by the hypoechogenic areas, it is not surprising that only minor changes in TUS were observed by Fisher et al. ${ }^{1}$ Apparently, the change in the area of the inflammatory infiltrates might not be measured directly by the change in percentage of hypoechogenic areas seen on ultrasound.

Second, another possible explanation why no more domains of the TUS change after rituximab treatment is that TUS is not sensitive enough to detect the histopathological changes. The consistency component is scored on a categorical scale, based on the ultrasonographers' estimate of the amount of hypoechogenic areas present. It is questionable whether the decrease in the glandular area occupied by infiltrating cells is reflected by a similar change in the ultrasound image. In other words, it is uncertain whether the applied scoring system (TUS) is sensitive enough to detect this change. With only a few categories, there has to be a relative large change in the amount of hypoechogenic areas present in the ultrasonographic image of a particular salivary gland to result in a different score. Furthermore, with regard to the ultrasonographic scoring, there are some other points to keep in mind. For example, ultrasound scoring was done live, but for an unbiased evaluation, blinded scoring is warranted. In addition, as already pointed out by Fisher et al, ${ }^{1}$ the ultrasound devices between the different centres were not standardised and intraobserver and interobserver reliability were not assessed. This last point is particularly important since previously there was more inconsistency in scoring the severity of the ultrasonographic characteristics ${ }^{10}$ as used in the study of Fisher et al. ${ }^{1}$ On the other hand, the ultrasonographers were able to reliably identify in which patients the ultrasonographic characteristics support the diagnosis of pSS. ${ }^{10}$

Finally, the most simple explanation for the minor TUS changes is that the TRACTISS study, a randomised double blind placebo controlled clinical TRial of Anti-B-Cell Therapy In patients with primary Sjögren's Syndrome, failed to meet its primary endpoint, that is, a change of $30 \%$ in oral dryness or fatigue on the visual analogue scale. ${ }^{8}$ Taking this into account, we should have been surprised if major differences in TUS were found. Another important point to mention is that in the TRACTISS study, ${ }^{8}$ the effect of rituximab treatment on the histopathology of the glandular tissue itself has not been studied, nor is any information given about how many patients had a positive salivary gland biopsy at the start of the study. Because of this, it is impossible to make a statement about whether or not the right subgroup of patients was included in the first place, to detect changes in TUS, as it appears that rituximab shows efficacy in a subgroup of patients with pSS. ${ }^{16}$ 
In conclusion, rituximab treatment leads to significant improvements in histopathological characteristics of salivary gland biopsies. This improvement was not reflected by a similar improvement in the amount of hypoechogenic areas identified with ultrasound by Fisher et al, ${ }^{1}$ but this could be due to several reasons, that is, ultrasound is not sensitive enough to detect the histopathological differences or does not reflect the changes seen in the biopsies and the TRACTISS study was a negative study, perhaps due to the chosen inclusion criteria. In order to make a statement about whether or not ultrasound is the right tool to measure treatment effect, the sensitivity to change should be determined in positive clinical trials. At this point, the baseline histopathological characteristics of the parotid gland should be used to predict the effect of rituximab therapy and to help us to determine a personalised treatment approach.

\section{Esther Mossel, ${ }^{1}$ Konstantina Delli, ${ }^{2}$ Suzanne Arends, ${ }^{1}$ Erlin A Haacke, ${ }^{1,3}$ Bert van der Vegt, ${ }^{3}$ Jolien F van Nimwegen, ${ }^{1}$ Alja J Stel, ${ }^{1}$ Fred K L Spijkervet, ${ }^{2}$ Arjan Vissink, ${ }^{2}$ Frans G M Kroese, ${ }^{1}$ Hendrika Bootsma ${ }^{1}$}

${ }^{1}$ Department of Rheumatology and Clinical Immunology, University Medical Center Groningen, Groningen, The Netherlands

${ }^{2}$ Department of Oral and Maxillofacial Surgery, University Medical Center Groningen, University of Groningen, Groningen, The Netherlands

${ }^{3}$ Department of Pathology and Medical Biology, University Medical Center

Groningen, University of Groningen, Groningen, The Netherlands

Correspondence to Dr Hendrika Bootsma, Department of Rheumatology and Clinical Immunology, University Medical Center Groningen, Groningen 9713, The Netherlands; h.bootsma@umcg.nl

Handling editor Josef S Smolen

Contributors The first manuscript was written by EM. All authors critically reviewed the letter and approved the final version to be published.

Competing interests None declared.

Patient consent Not required.

Provenance and peer review Not commissioned; internally peer reviewed.

(c) Article author(s) (or their employer(s) unless otherwise stated in the text of the article) 2019. All rights reserved. No commercial use is permitted unless otherwise expressly granted.

\section{Check for updates}

To cite Mossel E, Delli K, Arends S, et al. Ann Rheum Dis 2019;78:e27.

Received 1 March 2018

Accepted 1 March 2018

Published Online First 9 March 2018

\section{Linked}

- http://dx.doi.org/10.1136/annrheumdis-2018-213340

Ann Rheum Dis 2019;78:e27. doi:10.1136/annrheumdis-2018-213332

\section{REFERENCES}

1 Fisher BA, Everett CC, Rout J, et al. Effect of rituximab on a salivary gland ultrasound score in primary Sjögren's syndrome: results of the TRACTISS randomised double-blind multicentre substudy. Ann Rheum Dis 2018;77:412-6.

2 Delli K, Haacke EA, Kroese FG, et al. Towards personalised treatment in primary Sjögren's syndrome: baseline parotid histopathology predicts responsiveness to rituximab treatment. Ann Rheum Dis 2016;75:1933-8.

3 Delli K, Haacke EA, Kroese FG, et al. In primary Sjögren's syndrome high absolute numbers and proportions of $B$ cells in parotid glands predict responsiveness to rituximab as defined by ESSDAI, but not by SSRI. Ann Rheum Dis 2016;75:e34.

4 Cornec D, Costa S, Devauchelle-Pensec V, et al. Blood and salivary-gland BAFF-driven B-cell hyperactivity is associated to rituximab inefficacy in primary Sjögren's syndrome. J Autoimmun 2016;67:102-10.

5 Cornec D, Costa S, Devauchelle-Pensec V, et al. Do high numbers of salivary glandinfiltrating $B$ cells predict better or worse outcomes after rituximab in patients with primary Sjögren's syndrome? Ann Rheum Dis 2016;75:e33-8.

6 Pijpe J, Meijer JM, Bootsma H, et al. Clinical and histologic evidence of salivary gland restoration supports the efficacy of rituximab treatment in Sjögren's syndrome. Arthritis Rheum 2009:60:3251-6.

7 Haacke EA, van der Vegt B, Meiners PM, et al. Abatacept treatment of patients with primary Sjögren's syndrome results in a decrease of germinal centres in salivary gland tissue. Clin Exp Rheumatol 2017;35:317-20.

8 Bowman SJ, Everett CC, O'Dwyer JL, et al. Randomized controlled trial of rituximab and cost-effectiveness analysis in treating fatigue and oral dryness in primary Sjögren's Syndrome. Arthritis Rheumatol 2017;69:1440-50.

9 Jousse-Joulin S, Devauchelle-Pensec V, Cornec D, et al. Brief report: ultrasonographic assessment of salivary gland response to rituximab in primary Sjögren's Syndrome. Arthritis Rheumatol 2015;67:1623-8.

10 Delli K, Arends S, van Nimwegen JF, et al. Ultrasound of the major salivary glands is a reliable imaging technique in patients with clinically suspected primary Sjögren's syndrome. Ultraschall Med 2017 [EPub ahead of print 25 Oct 2017].

11 Jousse-Joulin S, Nowak E, Cornec D, et al. Salivary gland ultrasound abnormalities in primary Sjögren's syndrome: consensual US-SG core items definition and reliability. RMD Open 2017;3:e000364.

12 Mossel E, Arends S, van Nimwegen JF, et al. EULAR US-pSS study group. Scoring hypoechogenic areas in one parotid and one submandibular gland increases feasibility of ultrasound in primary Sjögren's syndrome. Ann Rheum Dis 2018;77:556-62.

13 Hocevar A, Ambrozic A, Rozman B, et al. Ultrasonographic changes of major salivary glands in primary Sjogren's syndrome. Diagnostic value of a novel scoring system. Rheumatology 2005;44:768-72.

14 Cornec D, Jousse-Joulin S, Costa S, et al. High-grade salivary-gland involvement, assessed by histology or ultrasonography, is associated with a poor response to a single rituximab course in primary Sjögren's Syndrome: data from the TEARS randomized trial. PLoS One 2016;11:e0162787.

15 Mossel E, Delli K, van Nimwegen JF, et al. The parotid gland connection: ultrasound and biopsies in primary Sjögren's syndrome. Ann Rheum Dis 2018;77:e38.

16 Bootsma H, Kroese FGM, Vissink A. Editorial: rituximab in the treatment of Sjögren's syndrome: is it the right or wrong drug? Arthritis Rheumatol 2017;69:1346-9. 